\title{
VARIATION STRUCTURES: RESULTS AND OPEN PROBLEMS
}

\author{
ANDRÁS NÉMETHI \\ Institute of the Romanian Academy, Bucharest \\ and \\ The Ohio State University, 231 West 18th Avenue, Columbus, Ohio 43210, U.S.A. \\ E-mail: nemethi@math.ohio-state.edu
}

1. Introduction. Studying the invariants of isolated hypersurface germs $f:\left(\mathbf{C}^{n+2}, 0\right)$ $\rightarrow(\mathbf{C}, 0)$, it is very useful to consider composed germs $f=p \circ \phi$, where $\phi:\left(\mathbf{C}^{n+2}, 0\right) \rightarrow$ $\left(\mathbf{C}^{2}, 0\right)$ has a manageable discriminant space (for example: $\phi$ is an isolated complete intersection singularity, for short ICIS $)$, and $p:\left(\mathbf{C}^{2}, 0\right) \rightarrow(\mathbf{C}, 0)$ is a curve singularity. This gives not only a very large class of examples with powerful testing role (for example, the germs of "generalized Sebastiani-Thom type", where $\phi(x, y)=(g(x), h(y))[9,10]$, or the topological series $f_{k}=p_{k} \circ \phi \rightarrow f_{\infty}=p_{\infty} \circ \phi$, when $\phi$ is an ICIS and $p_{k} \rightarrow p_{\infty}$ is a topological series $[10,17]$ of plane singularities), but also clarifies the most general case. To see this, complete the initial, arbitrary germ $f$ to an ICIS $(f, g)=\phi$ and take $p(c, d)=c$. If $g$ is a generic linear form then we recover the classical method of the polar curves, which is an effective inductive procedure.

In the composed case, the leading principle is the following: for a given invariant $i$, find a category $C(i)$ of supplementary structures ("of system of coefficients") defined either on $\left(\mathbf{C}^{2}, 0\right)$ or on the local complement of an analytic germ $\Delta \subset\left(\mathbf{C}^{2}, 0\right)$ (which, in general, is the discriminant space of $\phi$ ) with the following properties:

a) $\phi$ defines a structure $S(\phi)$ in $C(i)$, and

b) the invariant $i(f)$ can be computed in terms of the germ $p$ and the structure $S(\phi)$.

In this way, one expects that the computation of the invariant $i$ is reduced to lower dimensional topology (link topology of $p^{-1}(0) \cup \Delta$ ) with some representations, or to the study of the resolution graph of some plane singularities together with some special sheaf structures.

1991 Mathematics Subject Classification: Primary 32S50; Secondary 32S55.

Partially supported by the Netherlands Organisation for the Advancement of Scientific Research N.W.O.

The paper is in final form and no version of it will be published elsewhere. 
For example, if $i(f)=\zeta(f)=$ the zeta function of the germ $f$, then $C(\zeta)$ is the category of constructible complexes, and for any complex $S$ there is a natural definition of $i(f, S)=\zeta(f, S)$ which satisfies the above requirements, namely, for $S(\phi)=\mathbf{R} \phi_{*} \mathbf{C}_{\left(\mathbf{C}^{n+2}, 0\right)}$ one has $\zeta(f)=\zeta(p, S(\phi))[10]$.

If $i(f)=\operatorname{Spp}(f)=$ the spectral pairs of the germ $f$ (for the definition, see [18]), then $C(\mathrm{Spp})$ is the category of polarized mixed Hodge modules (for more details, see [15]).

The main object of this note is the category $C(\sigma)$ associated with the signature $\sigma(f)$ of the Milnor fiber of $f$.

Problem 1. Find the category $C(i)$ for other invariants.

For example, find a category $C(i)$ such that any ICIS $\phi$ (as above) gives an element $S(\phi) \in C(i)$. Moreover, define a "generalized Dynkin diagram" $D(p, S)$ for any (isolated) curve singularity $p$ and $S \in C(i)$ in such a way that $D(p, S(\phi))$ is the Dynkin diagram of $f=p \circ \phi$ (provided that $f$ has isolated singularity).

2. Variation structures. Definitions and examples. The needed category $C(i)$ in the case of the (equivariant) signature is the category of the variation structures $[11,13$, 14]. Notice that, in general, $C(i)$ is not uniquely determined by the properties a-b given in $\S 1$. Actually, the category of variation structures contains much additional topological information. Its definition follows.

If $U$ is a finite dimensional vector space then $U^{*}$ is its dual $\operatorname{Hom}_{\mathbf{C}}(U, \mathbf{C})$. There is a natural isomorphism $\theta: U \rightarrow U^{* *}$ given by $\theta(u)(\varphi)=\varphi(u)$. We denote the complex conjugation by $\bar{\imath}$. If $\varphi \in \operatorname{Hom}_{\mathbf{C}}\left(U, U^{\prime}\right)$, then $\bar{\varphi} \in \operatorname{Hom}_{\mathbf{C}}\left(U, U^{\prime}\right)$ is defined by $\bar{\varphi}(x):=\overline{\varphi(\bar{x})}$, and the dual $\varphi^{*}: U^{\prime *} \rightarrow U^{*}$ of $\varphi$ by $\varphi^{*}(\psi)=\psi \circ \varphi$.

A C-linear endomorphism $b: U \rightarrow U^{*}$ with $\overline{b^{*} \circ \theta}=\epsilon b(\epsilon= \pm 1)$ is called an $\epsilon$-hermitian form on $U$. The automorphisms $h: U \rightarrow U$ with $\bar{h}^{*} \circ b \circ h=b$ form the orthogonal group $\operatorname{Aut}(U ; b)$.

Definition. An $\epsilon$-hermitian isometric structure of the group $G$ is a system $\mathcal{I}=$ $(U ; b, \rho)$ such that $b$ is an $\epsilon$-hermitian non-degenerate form, and $\rho: G \rightarrow \operatorname{Aut}(U ; b)$ is a group endomorphism.

Any representation $\rho: G \rightarrow \operatorname{Aut}(U)$ defines a left action of $G$ on $\operatorname{Hom}\left(U^{*}, U\right)$ by $g * \varphi=\rho(g) \circ \varphi$. Then, by definition, a twisted-homeomorphism is a map $V: G \rightarrow$ $\operatorname{Hom}\left(U^{*}, U\right)$ with $V(g h)=\rho(g) \circ V(h)+V(g)$.

Definition. An $\epsilon$-hermitian variation structure of the group $G$ is a system $\mathcal{V}=$ $(U ; b, \rho, V)$ such that $b$ is an $\epsilon$-hermitian (maybe degenerate) form, $\rho$ is a representation of $G$ in $\operatorname{Aut}(U ; b), V$ is a twisted-homeomorphism, with respect to the left action of $G$ via $\rho$, and they satisfy the following compatibility conditions for any $g \in G$ :

(i) ${\overline{\theta^{-1} \circ V(g)}}^{*}=-\epsilon V(g) \circ \overline{\rho(g)}^{*}$, and

(ii) $V(g) \circ b=\rho(g)-I$.

Definition. Two $\epsilon$-hermitian variation structures $(U ; b, \rho, V)$ and $\left(U^{\prime} ; b^{\prime}, \rho^{\prime}, V^{\prime}\right)$ are isomorphic (denoted by $\approx$ ) if there exists a (C-linear) isomorphism $\varphi: U \rightarrow U^{\prime}$ such that $b=\bar{\varphi}^{*} b^{\prime} \varphi, \rho(g)=\varphi^{-1} \rho(g)^{\prime} \varphi$, and $V(g)=\varphi^{-1} V^{\prime}(g)\left(\bar{\varphi}^{*}\right)^{-1}$ for any $g \in G$. 
$H V_{\epsilon}(G)$ denotes the semigroup of isomorphism classes. (The semigroup structure is provided by the natural direct sum.)

Any base $\left\{e_{i}\right\}_{i}$ of $U$ defines a dual base $\left\{e_{i}^{*}\right\}_{i}$ of $U^{*}$ by $e_{j}^{*}\left(e_{i}\right)=1$ if $j=i$ and $=0$ otherwise. In all our matrix notations we will use the matrix representation in a convenient base and in its dual base.

EXAMPLES. 1. If $b$ is non-degenerate then $V(g)=(\rho(g)-I) b^{-1}$, i.e. the semigroup of non-degenerate variation structures is equivalent to the semigroup of $\epsilon$-hermitian isometric structures. We call these systems non-degenerate variation systems.

In general, the variation structures substitute the isometric structures in those cases when, from geometric considerations, the corresponding hermitian form is degenerate.

2. If $V(g)$ is an isomorphism, then $\rho(g)=-\epsilon V(g)\left(\overline{\theta^{-1} \circ V(g)^{*}}\right)^{-1}$, and $b=-V(g)^{-1}$ $\epsilon\left(\overline{\theta^{-1} \circ V(g)^{*}}\right)^{-1}$. In particular, if $G=\mathbf{Z}$, then the subsemigroup $H V_{\epsilon}^{s}(\mathbf{Z})=\{\mathcal{V} \subset$ $H V_{\epsilon}(\mathbf{Z}): V(1)$ an isomorphism $\}$ is equivalent to the semigroup of the sesquilinear forms over C. We call these systems simple.

3. If $\mathcal{V}_{i}=\left(U_{i} ; b_{i}, \rho_{i}, V_{i}\right)(i=1,2)$ are variation structures, then $\mathcal{V}_{1} \oplus \mathcal{V}_{2}=\left(U_{1} \oplus\right.$ $\left.U_{2} ; b_{1} \oplus b_{2}, \rho_{1} \oplus \rho_{2}, V_{1} \oplus V_{2}\right)$ is their direct sum in this category. The direct sum of $n$ copies of $\mathcal{V}$ is denoted by $n \mathcal{V}$. If $\mathcal{V}=(U ; b, \rho, V)$ then $-\mathcal{V}$ denotes $(U ;-b, \rho,-V)$ with the same $\epsilon$. The conjugate of $\mathcal{V}=(U ; b, \rho, V)$ is $\overline{\mathcal{V}}=(U ; \bar{b}, \bar{\rho}, \bar{V})$.

4. An important element in $H V_{\epsilon}^{s}(\mathbf{Z})$, provided by an isolated hypersurface singularity $f$, is $\mathcal{V}(f)$ generated by $(U ; b, \rho(1), V(1))=$ (middle homology of the Milnor fiber of $f$; intersection form, monodromy, variation map). Notice that the variation map $V(1)$ of $f$ can be identified with the inverse of the Seifert form (up to sign) [4], in particular, $V(1)$ is an isomorphism (cf. $\S 4$ ).

5. Consider an isolated complete intersection singularity $f:\left(\mathbf{C}^{n+2}, 0\right) \rightarrow\left(\mathbf{C}^{2}, 0\right)$ $(n>0)$. Let $\phi:(\mathcal{X}, 0) \rightarrow(S, 0)$ be a "good representative" of $f$ with discriminant locus $\Delta \subset S$. Consider a base-point $* \in S-\Delta$. The relative homology of the fiber $F=\phi^{-1}(*)$ is concentrated in $U=H_{n}(F, \mathbf{C})$. Identify its dual $U^{*}$ with $H_{n}(F, \partial F ; \mathbf{C})$, and extend the real intersection form to a hermitian form $b: U \rightarrow U^{*}$. The monodromy representation $\rho: G=\pi_{1}(S-\Delta, *) \rightarrow \operatorname{Aut}(U ; b)$, and the variation map $V: G \rightarrow \operatorname{Hom}\left(U^{*}, U\right)$ constitute a system $\mathcal{V}(\phi)=(U ; b, \rho, V)$ which is our basic example of $(-1)^{n}$-hermitian variation structure.

In the following examples $G=\mathbf{Z}$ and $V(1)=V, \rho(1)=h$ and we will use the notation $\epsilon=(-1)^{n}$. We denote by $J_{k}$ the $k \times k$ Jordan block

$$
\left(\begin{array}{cccc}
1 & 1 & & \\
& 1 & \ddots & \\
& & \ddots & 1 \\
& & & 1
\end{array}\right) .
$$

6. Consider $\lambda \in \mathbf{C}^{*}-S^{1}$. The $\epsilon$-HV $\mathcal{V}^{2 k}(\lambda)$ is defined by

$$
\mathcal{V}_{\lambda}^{2 k}=\left(\mathbf{C}^{2 k} ;\left(\begin{array}{cc}
0 & I \\
\epsilon I & 0
\end{array}\right),\left(\begin{array}{cc}
\lambda J_{k} & 0 \\
0 & \frac{1}{\lambda} J_{k}^{*,-1}
\end{array}\right),\left(\begin{array}{cc}
0 & \epsilon\left(\lambda J_{k}-I\right) \\
\frac{1}{\lambda} J_{k}^{*,-1}-I & 0
\end{array}\right)\right) .
$$

Note that $\mathcal{V}_{\lambda}^{2 k} \approx \mathcal{V}_{1 / \bar{\lambda}}^{2 k} \approx-\mathcal{V}_{\lambda}^{2 k}$. 
7. We are looking for a non-degenerate $k \times k$ matrix $b$ such that $\bar{b}^{*}=\epsilon b$ and $J_{k}^{*} b J_{k}=$ $b$. It is immediate that $b_{i j}=0$ if $i+j \leq k$ and $b_{k+1-i, i}=(-1)^{i+1} b_{k, 1}$. By [8] the isomorphism class of $\left(b, J_{k}\right)$ is determined by $b_{k, 1}$. Since $b$ is non-degenerate, $b_{k, 1} \neq 0$. Since for any $t \in(0, \infty)$ one has $\left(U ; b, J_{k}, V\right) \approx\left(U ; t^{2} b, J_{k}, t^{-2} V\right)$, we can assume that $b_{k, 1}=\omega \in S^{1}$. By the hermitian property of $b$ one has $\bar{\omega}=\epsilon(-1)^{k-1} \omega$. This equation has two solutions. In conclusion, there are exactly two non-degenerate forms $b=b_{ \pm}^{k}$ (up to isomorphism) with $\bar{b}^{*}=\epsilon b$ and $J_{k}^{*} b J_{k}=b$. Their representatives are chosen so that $\left(b_{ \pm}^{k}\right)_{k, 1}= \pm i^{-n^{2}-k+1}$ (this strange choice has a Hodge-theoretical motivation, cf. §4). Note that $b_{k, 1}=B\left(e_{k}, e_{1}\right)=B\left(e_{k},\left(J_{k}-I\right)^{k-1} e_{k}\right)=B\left(e_{k},\left(\log J_{k}\right)^{k-1} e_{k}\right)$. (Here $\left\{e_{l}\right\}_{l}$ denotes the standard base of $\mathbf{C}^{k}$.)

Let $\lambda \in S^{1}$. If $h=\lambda J_{k}$, then by the above argument, there are exactly two nondegenerate $\epsilon$-HVS's (up to isomorphism):

$$
\mathcal{V}_{\lambda}^{k}( \pm 1)=\left(\mathbf{C}^{k} ; b_{ \pm}^{k}, \lambda J_{k},\left(\lambda J_{k}-I\right)\left(b_{ \pm}^{k}\right)^{-1}\right)
$$

where $\omega=\left(b_{ \pm}^{k}\right)_{k, 1}= \pm i^{-n^{2}-k+1}$.

If $\lambda \neq 1$, then any HVS with $h=\lambda J_{k}$ is non-degenerate. If $h=J_{k}$, then there are some degenerate structures, too.

8. Suppose that $k \geq 2$ and $h=J_{k}$ but $b$ is degenerate. Since $\operatorname{ker} b \subset \operatorname{ker}(h-I)$, and $\operatorname{dim} \operatorname{ker}\left(J_{k}-I\right)=1$, one has $\operatorname{ker} b=\operatorname{ker}(h-I)$. Similarly as above, any degenerated form $b$ with $\operatorname{ker} b=\operatorname{ker}\left(J_{k}-I\right)$ and $\bar{b}^{*}=\epsilon b$ and $\bar{h}^{*} b h=b$ has the properties $b_{i, j}=0$ if $i+j \leq k+1$, and $b_{k+2-i, i}=(-1)^{i} b_{k, 2}$. Therefore $b_{k, 2} \neq 0$ and in the isomorphism class of the structure there is a representative with $b_{k, 2}=\omega \in S^{1}$. By symmetry, $\bar{\omega}=(-1)^{n+k} \omega$ and $b$ is completely determined by $b_{k, 2}$ modulo an isomorphism. So, we have exactly two solutions $\tilde{b}_{ \pm}^{k}$ (up to isomorphism) with $\left(\tilde{b}_{ \pm}^{k}\right)_{k, 2}= \pm(-1)^{n+1} i^{-(n+1)^{2}-k+1}$. Moreover, $V$ is completely determined by $h$ and $b$ (up to isomorphism). Therefore there are exactly two degenerate structures with $h=J_{k}$ and $k \geq 2$ :

$$
\tilde{\mathcal{V}}_{1}^{k}( \pm 1)=\left(\mathbf{C}^{k} ; \tilde{b}_{ \pm}^{k}, J_{k}, \tilde{V}_{ \pm}^{k}\right)
$$

where $\left(\tilde{b}_{ \pm}^{k}\right)_{k, 2}=B_{ \pm}^{k}\left(e_{k},\left(\log J_{k}\right)^{k-2} e_{k}\right)= \pm(-1)^{n+1} i^{-(n+1)^{2}-k+1}= \pm i^{-n^{2}-k+2}$. In fact,

$$
b=\tilde{b}_{ \pm}^{k}=\left(\begin{array}{cc}
0 & 0 \\
0 & b_{ \pm}^{k-1}
\end{array}\right) .
$$

Note that the structure can also be recognized from $\left(\left(\tilde{V}_{ \pm}^{k}\right)^{-1}\right)_{k, 1}= \pm i^{-n^{2}-k+2}$.

By computation we get that $\tilde{V}_{ \pm}^{k}$ is an isomorphism. In particular, the variation structures $\mathcal{V}_{\lambda}^{k}( \pm 1)$, where $\lambda \in S^{1}-\{1\}$ resp. $k \geq 1$, and $\tilde{\mathcal{V}}_{1}^{k}( \pm 1)$ where $k \geq 2$, are simple. They are determined by the corresponding isometric structures $\left(\mathbf{C}^{k} ; b, h\right)$.

9. Suppose that $U=\mathbf{C}$ and $h=1_{\mathbf{C}}$. Then there are exactly five HVS's (up to isomorphism):

$$
\mathcal{V}_{1}^{1}( \pm 1)=\left(\mathbf{C} ; \pm i^{-n^{2}}, 1_{\mathbf{C}}, 0\right), \quad \tilde{\mathcal{V}}_{1}^{1}( \pm 1)=\left(\mathbf{C} ; 0,1_{\mathbf{C}}, \pm i^{n^{2}-1}\right),
$$

and

$$
\mathcal{T}=\left(\mathbf{C} ; 0,1_{\mathbf{C}}, 0\right)
$$

Note that in $\tilde{\mathcal{V}}_{1}^{1}( \pm 1)$ the variation structure is not determined by its underlying isometric structure. 
10. In order to unify the notations of the simple structures, we introduce $\mathcal{W}_{\lambda}^{k}( \pm 1)=$ $\mathcal{V}_{\lambda}^{k}( \pm 1)$ if $\lambda \in S^{1}-\{1\}$, and $=\tilde{\mathcal{V}}_{1}^{k}( \pm 1)$ if $\lambda=1$. Set $s=1$ if $\lambda=1$ and $=0$ otherwise. Then $\overline{\mathcal{W}}_{\lambda}^{k}( \pm 1)=\mathcal{W} \frac{k}{\lambda}\left( \pm(-1)^{-n^{2}-k+1+s}\right)$.

11. Consider the following matrices:

$$
\mathbf{b}=\left(\begin{array}{ccc}
0 & 0 & 1 \\
0 & 0 & 0 \\
1 & 0 & 1
\end{array}\right), \quad \mathbf{h}=\left(\begin{array}{ccc}
1 & 0 & 0 \\
0 & 1 & 1 \\
0 & 0 & 1
\end{array}\right), \quad \mathbf{V}=\left(\begin{array}{ccc}
0 & -1 & 0 \\
1 & 0 & 0 \\
0 & 0 & 0
\end{array}\right)
$$

They define an indecomposable (+1)-HVS, but the automorphism $h$ has two Jordan blocks. Note that even the associated (degenerate) isometric structure $(b, h)$ is indecomposable.

If $\mathcal{V}_{i}(i=1,2)$ are simple $\epsilon_{i}$-hermitian variation structures, then the tensor product $V_{1} \otimes V_{2}$ defines a new simple $\epsilon$-structure (for any $\epsilon$ ). The corresponding automorphisms are related by ${ }^{\otimes} h=-\epsilon \epsilon_{1} \epsilon_{2} h_{1} \otimes h_{2}$. If we want to emphasize the sign of $\epsilon$ in the tensor product, we write $\mathcal{V}_{1} \otimes_{\varepsilon} \mathcal{V}_{2}$. For the ring structure of $H V_{\epsilon}^{s}(\mathbf{Z})$, see [12].

PROBLEM 2. Find the tensor product of two variation structures.

3. Classification. In this section $G=\mathbf{Z}$ and the system $\mathcal{V}=(U ; b, h, V)$ denotes $h=\rho(1)$ and $V=V(1)$.

First we recall (the complex version of) Milnor's result [8] (see also [16]):

Any isometric structure $(U ; b, h)$ is a sum of indecomposable ones. The indecomposable structures are the corresponding isometric structures of $\mathcal{V}_{\lambda}^{k}( \pm 1)$, where $\lambda \in S^{1}$, and of $\mathcal{V}_{\lambda}^{2 k}$, where $\lambda \in \mathbf{C}^{*}-S^{1}$.

On the other hand, the following holds:

TheOREm ([11]). (a) An $\epsilon$-hermitian variation structure is uniquely expressible as a direct sum $\mathcal{V}^{\prime} \oplus \mathcal{V}^{\prime \prime}$ so that $h^{\prime}-I$ is an isomorphism (in particular, $\mathcal{V}^{\prime}$ is simple and non-degenerate), and $h^{\prime \prime}-I$ is nilpotent.

(b) A simple $\epsilon$-hermitian variation structure is uniquely expressible as a sum of indecomposable ones up to order of summands and isomorphism. The indecomposable structures are

$$
\begin{gathered}
\mathcal{W}_{\lambda}^{k}( \pm 1) \quad \text { where } k \geq 1, \lambda \in S^{1}, \text { and } \\
\mathcal{V}_{\lambda}^{2 k} \quad \text { where } k \geq 1,0<|\lambda|<1 .
\end{gathered}
$$

R e m a r k. Part (b) of this theorem gives a classification of comlex sesquilinear forms (with respect to complex conjugation) over finite dimensional $\mathbf{C}$-vector spaces.

If two real non-degenerate bilinear forms are isomorphic as sesquilinear forms over $\mathbf{C}$, then they are isomorphic as real bilinear forms. In particular, the study of real simple variation structures is equivalent to the study of the complex ones.

Problem 3. Classify the variation structures for $G=\mathbf{Z}$ (cf. Example 11).

Classify a class of variation structures which includes the structures given by hypersurface singularities with one-dimensional singular locus. 
Problem 4a. Any hypersurface isolated singularity gives a simple variation structure ( of $G=\mathbf{Z}$ ) (cf. Example 3). Find natural restrictions provided by the singularity theory, and classify variation structures with the corresponding restrictions.

4b. Let $\phi$ be an ICIS with two-dimensional base space (as in the introduction). Let $(U, E) \rightarrow(B, \Delta)$ be an embedded resolution of its discriminant locus. Let $\Delta_{0}$ be one of the irreducible components of $\Delta, \tilde{\Delta_{0}}$ its strict transform, and finally $P=\tilde{\Delta_{0}} \cap E$. Let $U_{P}$ be a small neighbourhood of the point $P$ in $U$. The variation structure $\mathcal{V}(\phi)$ restricted to the subgroup $\mathbf{Z}^{2}=\pi_{1}\left(U_{P} \backslash E\right)$ gives a variation structure of the group $G=\mathbf{Z}^{2}$. Now, find a natural restriction which is provided by this geometric situation and classify variation structures of the group $G$ with this restriction.

4. Variation structures and isolated hypersurface singularities. Consider an isolated hypersurface singularity $f:\left(\mathbf{C}^{n+1}, 0\right) \rightarrow(\mathbf{C}, 0)$. We recall the definitions of the main invariants.

For $\epsilon$ sufficiently small and $0<\delta \ll \epsilon$ define $S_{\delta}^{1}=\{w: \| w \mid=\delta\} \subset \mathbf{C}$ and $E:=f^{-1}\left(S_{\delta}^{1}\right) \cap\{z:|z| \leq \epsilon\} \subset \mathbf{C}^{n+1}$. Then the induced map $f:(E, \partial E) \rightarrow S_{\delta}^{1}$ is a locally trivial fibration with fiber $(F, \partial F)$, such that $f \mid \partial E$ is trivial. The (Milnor) fiber $F$ is homotopically equivalent to a bouquet $\bigvee S^{n}$, therefore its reduced (real) homology (cohomology) is concentrated in $U_{\mathbf{R}}=\tilde{H}_{n}(F, \mathbf{R})\left(U_{\mathbf{R}}^{*}=\tilde{H}^{n}(F, \mathbf{R})\right)$. The characteristic map of the above fibration at (co)homological level defines the algebraic monodromies $h_{\mathbf{R}}: U_{\mathbf{R}} \rightarrow U_{\mathbf{R}}$ and $T_{\mathbf{R}}=h_{\mathbf{R}}^{*,-1}: U_{\mathbf{R}}^{*} \rightarrow U_{\mathbf{R}}^{*}$. The natural, real intersection form is denoted by $b_{\mathbf{R}}: U_{\mathbf{R}} \rightarrow U_{\mathbf{R}}^{*}$. Fixing a trivialization of $f \mid \partial E$ one defines a variation map $\operatorname{Var}: U_{\mathbf{R}}^{*} \rightarrow U_{\mathbf{R}}$.

These invariants satisfy the relations: $\operatorname{Var} \circ b_{\mathbf{R}}=h_{\mathbf{R}}-I, h_{\mathbf{R}}^{*} \circ b_{\mathbf{R}} \circ h_{\mathbf{R}}=b_{\mathbf{R}}, b_{\mathbf{R}}^{*} \circ \theta=\epsilon b_{\mathbf{R}}$, and $\operatorname{Var}^{*}=-\epsilon \operatorname{Var} \circ h_{\mathbf{R}}^{*}$, where $\epsilon=(-1)^{n}$.

In particular, the complex maps $b=b_{\mathbf{R}} \otimes 1_{\mathbf{C}}, h=h_{\mathbf{R}} \otimes 1_{\mathbf{C}}$, and $V=\operatorname{Var} \otimes 1_{\mathbf{C}}$ define a $(-1)^{n}$-HVS on $U=U_{\mathbf{R}} \otimes \mathbf{C}$. It is denoted by $\mathcal{V}(f)$. (Notice that here $b$ is a hermitian form rather than a bilinear form.)

Recall that $V$ is an isomorphism (cf. Example 4), therefore our variation structure is simple. The real Seifert form $L$ can be defined as follows. If $\langle$,$\rangle denotes the pairing between$ $H_{n}(F, \partial F, \mathbf{R})$ and $H_{n}(F, \mathbf{R})$, then for $a, b \in H_{n}(F, \mathbf{R})$ one has $L(a, b):=\left\langle\operatorname{Var}^{-1}(a), b\right\rangle$. By our notation, $\langle$,$\rangle identifies H_{n}(F, \partial F, \mathbf{R})$ with $U^{*}$, therefore Var can be identified with the inverse of the Seifert form (cf. [4] or [1, p. 41]).

Consider the Jordan decompositions $T=T_{s} T_{u}$ and the generalized eigenspaces $U_{\lambda}^{*}=$ $\operatorname{ker}\left(T_{s}-\lambda I\right)$. Set $\log T_{u}=N=\bigoplus N_{\lambda}$. Let $s=0$ if $\lambda \neq 1$, and $=1$ if $\lambda=1$.

The space $U_{\lambda}^{*}$ carries a mixed Hodge structure with weight filtration centered at $n+s$. For $r \geq 0$, the space

$$
P_{r, \lambda}=\operatorname{ker}\left(N_{\lambda}^{r+1}: \mathrm{Gr}_{n+s+r}^{W} U_{\lambda}^{*} \rightarrow \operatorname{Gr}_{n+s-r-2}^{W} U_{\lambda}^{*}\right)
$$

carries an induced Hodge structure of weight $n+s+r$ :

$$
P_{r, \lambda}=\bigoplus_{a+b=n+s+r} P_{\lambda}^{a, b} .
$$

By the monodromy therem $a+b=n+s+r \leq 2 n$. 
The discrete invariants of the Hodge and the weight filtration are collected in the Hodge numbers: $h_{\lambda}^{p, q}=\operatorname{dim} \operatorname{Gr}_{F}^{p} \operatorname{Gr}_{p+q}^{W} U_{\lambda}^{*}$, or equivalently, in the dimensions of the primitive spaces $p_{\lambda}^{a, b}=\operatorname{dim} P_{\lambda}^{a, b}(r=a+b-n-s \geq 0)$. Since $N_{\lambda}$ is a morphism of Hodge structures of type $(-1,-1)$, one has

$$
\begin{gathered}
p_{\lambda}^{a, b}=h_{\lambda}^{a, b}-h_{\lambda}^{a+1, b+1} \quad(r=a+b-n-s \geq 0), \quad \text { and } \\
h_{\lambda}^{a, b}=\sum_{l \geq 0} p_{\lambda}^{a+l, b+l} \quad(a+b \geq n+s) .
\end{gathered}
$$

The connection between the topological invariant $\mathcal{V}(f)$ and the Hodge theoretical invariants $p_{\lambda}^{a, b}(f)$ is given in the following

THEOREM ([11]).

$$
\mathcal{V}(f)=\bigoplus_{\lambda} \bigoplus_{2 n \geq a+b \geq n+s} p_{\lambda}^{a, b}(f) \mathcal{W}_{\lambda}^{r+1}\left((-1)^{b}\right)
$$

where $s=0$ if $\lambda \neq 1, s=1$ if $\lambda=1$ and $r=a+b-n-s \geq 0$. In particular, the Hodge numbers determine the real Seifert form.

This result is similar to the Hodge signature theorem in the case of smooth projective varieties. In that classical case, the signature is given by the collapsed $\mathbf{Z}_{2}$ ("even-odd") Hodge decomposition, where the collapse is induced by the polarization. In our case, the real Seifert form of an isolated singularity is equivalent to the collapsed mixed Hodge structure associated with the singularity.

EXAMPLE. Let $f: \mathbf{C}^{n+1} \rightarrow \mathbf{C}$ be a quasi-homogeneous polynomial of type $\left(w_{0}, \ldots, w_{n}\right)$ with isolated singularity at the origin. Let $\left\{z^{\alpha}: \alpha \in \mathcal{I} \subset \mathbf{N}^{n+1}\right\}$ be a set of monomials in $\mathbf{C}[z]$ whose residue classes form a basis for the Milnor algebra $\mathbf{C}[[z]] /(\partial f)$. For $\alpha \in \mathcal{I}$ let $l(\alpha)=\sum_{i=0}^{n}\left(\alpha_{i}+1\right) w_{i}$. Then

$$
\mathcal{V}(f)=\bigoplus_{\alpha \in \mathcal{I}} \mathcal{W}_{\exp (2 \pi i l(\alpha))}^{1}\left((-1)^{[l(\alpha)]}\right),
$$

where $[\cdot]$ denotes the integral part.

Problem 5. In the context of Problem 4b there is a natural limit mixed Hodge structure with the action of $\mathbf{Z}^{2}$ (via the semisimple part of the monodromy representation). Extend the above Theorem to this case.

To end this section we discuss some properties of variation structures which are satisfied by the Seifert form of the isolated singularities.

Let $\# \mathcal{V}$ be the number of $\mathcal{V}$-components in $\mathcal{V}(f)$.

There are several obstructions to the decomposition of $\mathcal{V}(f)$. The first is the stability of $\mathcal{V}(f)$ with respect to the complex conjugation:

$$
\# \mathcal{W}_{\lambda}^{r+1}( \pm 1)=\sum_{(-1)^{b}= \pm 1} p_{\lambda}^{a, b}=\sum_{(-1)^{a}= \pm(-1)^{n+r+s}} p_{\bar{\lambda}}^{b, a}=\# \mathcal{W}_{\bar{\lambda}}^{r+1}\left( \pm(-1)^{n+r+s}\right)
$$

(where $a+b-n-s=r$ ). 
Now, since $a \leq n$ and $b \leq n, \mathcal{V}(f)$ determines the numbers $p_{\lambda}^{a, b}$ where $(a, b)=$ $(n, n),(n, n-1),(n-1, n)$ and $(n-1, n-1)$ (with $a+b \geq n+s$.$) For these pairs,$

$$
p_{\lambda}^{a, b}=\# \mathcal{W}_{\lambda}^{a+b-n-s+1}\left((-1)^{b}\right) \text {. }
$$

In particular, for $n=1$, the system of Hodge numbers is completely determined by $\mathcal{V}(f)$. For $n=2$, only $\left\{p_{1}^{a, b}\right\}_{a, b} ; p_{\lambda}^{2,2} ; p_{\lambda}^{2,1} ; p_{\lambda}^{1,2} ; p_{\lambda}^{1,1}$ and the sum $p_{\lambda}^{0,2}+p_{\lambda}^{2,0}=\# \mathcal{V}_{\lambda}^{1}(+1)$ are determined by $\mathcal{V}(f)$.

The above relation gives:

Proposition ([11]). The structures $\mathcal{W}_{\lambda}^{n+1-s}\left((-1)^{n+1}\right)$ do not appear in the decomposition of $\mathcal{V}(f)$ for any isolated singularity $f:\left(\mathbf{C}^{n+1}, 0\right) \rightarrow(\mathbf{C}, 0)$.

This obstruction is nontrivial even for $n=1: \mathcal{V}_{\lambda}^{2}(+1), \lambda \neq 1$ and $\tilde{\mathcal{V}}_{1}^{1}(+1)$ cannot be components of an algebraic Seifert form. Both cases $(n=1 ; s=0$ and $s=1)$ were proved by Neumann [16] using the splice geometry of curve singularities.

Another property which is satisfied by simple variation structures provided by isolated hypersurface singularities is the following [5]:

Proposition. If $f:\left(\mathbf{C}^{n+1}, 0\right) \rightarrow(\mathbf{C}, 0)$ is an isolated singularity such that its monodromy $h$ has a Jordan block of size $n+1$ (necessarily for an eigenvalue $\neq 1)$, then $h$ has a Jordan block of size $n$ for the eigenvalue $=1$.

Problem 6. For a fixed $n$, find the complete set of simple variation structures $(G=\mathbf{Z})$ which are given by isolated hypersurface singularities.

\section{The Witt group of variation structures $([14])$}

Definition. A hermitian variation structure is hyperbolic if there exists a kernel $K \subset U$, i.e. a subset $K$ such that

(a) $\operatorname{dim} K=\frac{1}{2} \operatorname{dim} U$,

(b) $K \subset K^{\perp}=\{x: B(x, y)=0$ for any $y \in K\}$,

(c) For any $g \in G$ one has $\rho(g)(K) \subset K$ and $V(g)\left(K^{*}\right) \subset K$ where $K^{*}=\left\{\varphi \in U^{*}\right.$ : $\bar{\varphi}(K)=0\}$.

EXAMPLES. 1. If $b$ is non-degenerate then $\mathcal{V}$ is hyperbolic if and only if the isometric structure $(U ; b, \rho)$ is hyperbolic (i.e. there exists a $\rho$-invariant $K$ with $K=K^{\perp}$ ).

2. Consider $-\mathcal{V}=(U ;-b, \rho,-V)$. Then $\mathcal{V} \oplus(-\mathcal{V})$ is hyperbolic with kernel $K=\Delta U=$ $\{(x, x) \mid x \in U\}$.

In particular, the semigroup $W V_{\epsilon}(G)=\left(H V_{\epsilon}(G) /\{\right.$ hyperbolic structures $\left.\}, \oplus\right)$ is actually a group. It is called the Witt group of the variation structures of $G$.

For any $\chi \in \operatorname{Hom}\left(\mathbf{Z}^{k}, \mathbf{C}^{*}\right)$, we define the generalized eigenspace $U_{\chi}=\{x \in U$ : $(\rho(g)-\chi(g))^{N} x=0$ for some $N$ and any $\left.g \in G\right\} . \hat{G}=\operatorname{Hom}\left(\mathbf{Z}^{k}, S^{1}\right)$ denotes the group of characters.

Theorem. Let $G=\mathbf{Z}^{k}$. Then:

(a) There is a direct sum decomposition

$$
(U ; b, \rho, V)=\left(U^{\prime} ; b^{\prime}, \rho^{\prime}, V^{\prime}\right) \oplus \bigoplus_{\chi \in \hat{G}}\left(U_{\chi} ; b_{\chi}, \rho_{\chi}, V_{\chi}\right)
$$


where $U^{\prime}=\oplus_{\chi \notin \hat{G}} U_{\chi}$. Moreover, $\left(U^{\prime} ; b^{\prime}, \rho^{\prime}, V^{\prime}\right)$ is hyperbolic.

(b) $(U ; b, \rho, V)$ is hyperbolic if and only if $\left(U_{\chi} ; b_{\chi}, \rho_{\chi}, V_{\chi}\right)$ is hyperbolic for any $\chi \in \hat{G}$, in particular

$$
W V_{\epsilon}(G)=\bigoplus_{\chi \in \hat{G}} W V_{\epsilon}(G)_{\chi}
$$

Here $W V_{\epsilon}(G)_{\chi}$ is the Witt group of variation structures $\mathcal{V}$ with $\rho(g)-\chi(g) I$ nilpotent for any $g \in G$.

(c)

$$
W V_{\epsilon}\left(\mathbf{Z}^{k}\right)_{\chi}= \begin{cases}\mathbf{Z} & \text { if } \chi \in \hat{G}-\{1\} \\ \mathbf{Z}_{2} & \text { if } \chi=1\end{cases}
$$

The generators are $\left(\mathbf{C} ; \pm i^{(1-\epsilon) / 2}, \chi, \pm(\chi-1) i^{(\epsilon-1) / 2}\right)$ if $\chi \neq 1$, and $(\mathbf{C} ; 0,1,0)$ if $\chi=1$.

Problem 7. Extend the above classification to more general groups. (It would be ideal to have a classification theorem for the local fundamental groups of complements of curve singularities $p^{-1}(0) \subset\left(\mathbf{C}^{2}, 0\right)$.)

6. Wall's cocycle associated with a variation structure ([14]). Let $\mathcal{V}=$ $(U ; b, \rho, V)$ be an $\epsilon$-hermitian variation structure of $G$. Then $b$ defines an $\epsilon$-hermitian non-degenerate form $\Phi$ on $U^{*} \oplus U$ by

$$
\Phi((\varphi, u),(\psi, v))=\epsilon \bar{\psi}(u)+\varphi(\bar{v})+b(u)(\bar{v}) .
$$

Any $g \in G$ defines two maps $s_{r}(g), s_{l}(g): U^{*} \oplus U \rightarrow U^{*} \oplus U$ defined by

$$
\begin{aligned}
& s_{l}(g)(\varphi, u)=\left(\varphi, \rho(g) u-\rho(g) V\left(g^{-1}\right) \varphi\right), \\
& \left.s_{r}(g)(\varphi, u)=(\overline{\rho(g)})^{*,-1} \varphi,-V(g) \varphi+u\right) .
\end{aligned}
$$

Then $s_{l}$ resp. $s_{r}$ are representations of the group $G$ in the orthogonal group $O(\Phi)$ of $\Phi$.

For any $g \in G$, define $K_{g}=\left\{(\varphi, u) \in U^{*} \oplus U: V(g) \varphi=u\right\}$. It is not hard to verify that $K_{g}$ is a $\Phi$-kernel, i.e. $K_{g}=K_{g}^{\perp}$ (the latter one is the $\Phi$-orthogonal). Moreover, for any $g$ and $h$,

$$
s_{l}(h) K_{g}=K_{h g} \quad \text { and } \quad s_{r}(h) K_{g}=K_{g h^{-1}} .
$$

Any three kernels $K_{i}(i=1,2,3)$ in $U^{*} \oplus U$ define an $(-\epsilon)$-hermitian form [19]. We recall this construction. Set $K_{1} \cap\left(K_{2}+K_{3}\right)=\left\{x_{1} \in K_{1}\right.$ : there exist $x_{2} \in K_{2}, x_{3} \in K_{3}$ with $\left.x_{1}+x_{2}+x_{3}=0\right\}$. On this space define the sesquilinear form $\Psi\left(x_{1}, x_{1}^{\prime}\right)=\Phi\left(x_{1}, x_{2}^{\prime}\right)$ (where $x_{1}^{\prime}+x_{2}^{\prime}+x_{3}^{\prime}=0$ and $x_{2}^{\prime} \in K_{2}, x_{3}^{\prime} \in K_{3}$ ). Then $\Psi$ is $(-\epsilon)$-hermitian with kernel Ker $=K_{1} \cap K_{2}+K_{1} \cap K_{3}$. We define $\sigma\left(\mathcal{V} ; K_{1}, K_{2}, K_{3}\right)$ as the signature of the induced nondegenerate form on $K_{1,2,3}=K_{1} \cap\left(K_{2}+K_{3}\right) /$ Ker multiplied by $\epsilon$ (if there is no danger of confusion then it is denoted by $\left.\sigma\left(K_{1}, K_{2}, K_{3}\right)\right)$. If $K_{i}=K_{j}$ for some pair $(i, j)$, then $K_{1,2,3}=0$, hence $\sigma\left(K_{1}, K_{2}, K_{3}\right)=0$.

Lemma. (a) $\sigma\left(K_{\tau(1)}, K_{\tau(2)}, K_{\tau(3)}\right)=\operatorname{sign}(\tau) \sigma\left(K_{1}, K_{2}, K_{3}\right)$ for any permutation $\tau \in$ $\mathcal{S}_{3}$ (here $\operatorname{sign}(\tau) \in\{ \pm 1\}$ is the sign of $\tau$ ).

(b) $\sigma\left(K_{1}, K_{2}, K_{3}\right)-\sigma\left(K_{0}, K_{2}, K_{3}\right)+\sigma\left(K_{0}, K_{1}, K_{3}\right)-\sigma\left(K_{0}, K_{1}, K_{2}\right)=0$ for any kernels $K_{i}(i=1,2,3,4)$. 
(c) If $o \in O(\Phi)$ is an orthogonal automorphism, then $\sigma\left(o\left(K_{1}\right), o\left(K_{2}\right), o\left(K_{3}\right)\right)=$ $\sigma\left(K_{1}, K_{2}, K_{3}\right)$. In particular,

$$
\sigma\left(K_{h g_{1}}, K_{h g_{2}}, K_{h g_{3}}\right)=\sigma\left(K_{g_{1} h}, K_{g_{2} h}, K_{g_{3} h}\right)=\sigma\left(K_{g_{1}}, K_{g_{2}}, K_{g_{3}}\right) .
$$

Therefore $\sigma\left(\mathcal{V} ; K_{1}, K_{2}, K_{3}\right)$ defines a homogeneous cocycle of the group $G$ in $\mathbf{Z}(\mathbf{Z}$ is considered with the trivial $G$-action). The corresponding non-homogeneous cocycle is $\sigma(g, h)=\sigma\left(K_{e}, K_{g}, K_{g h}\right)$ ( $e$ is the neutral element of $\left.G\right)$. This cocycle is a coboundary if there exists a function $f: G \rightarrow \mathbf{Z}$ such that $\sigma(g, h)=f(g)+f(h)-f(g h)$; i.e. if $\sigma\left(K_{e}, K_{g}, K_{h}\right)=\delta(f)=f(g)+f\left(g^{-1} h\right)-f(h)$. The semigroup morphism

$$
c_{1}: H V_{\epsilon}(G) \rightarrow H^{2}(G, \mathbf{Z}), \quad c_{1}(\mathcal{V})=\sigma(\mathcal{V} ; \cdot, \cdot, \cdot)
$$

is not trivial in general. For example, if $G$ is the mapping class group $\Gamma_{g}$ and

$$
\mathcal{V}=\left(\mathbf{C}^{2 g} ;\left(\begin{array}{cc}
0 & I \\
-I & 0
\end{array}\right), \rho,(\rho-I) b^{-1}\right)
$$

where $\rho$ is given by $\Gamma_{g} \rightarrow S p(2 g, \mathbf{C})$ ("passing to homology"), then $c_{1}(\mathcal{V})$ is the generator of $H^{2}(G, \mathbf{Z})=\mathbf{Z}[6]$.

Remark. Set $B=\left\{z \in \mathbf{C}^{2}:|z| \leq 2 ;|z-1| \geq 1 / 2 ;|z+1| \geq 1 / 2\right\}$. Let $(E, \partial E) \stackrel{p}{\rightarrow} B$ be a $C^{\infty}$ fiber bundle of a pair of spaces with fiber $(F, \partial F)$ such that the induced bundle $\partial F \rightarrow \partial E \stackrel{p}{\rightarrow} B$ is trivial. Assume that $\operatorname{dim} F=4 k-2(k \geq 1)$. The fibration $p$ defines a variation structure $\mathcal{V}(p)$ of the free group (with two generators) $G=\pi_{1}(B)$ in a natural way (similarly to Example 5). By Wall's theorem [19] (see also [7]) the signature $\sigma(E)$ is $\sigma\left(K_{e}, K_{g}, K_{h}\right)$, where $g$ and $h$ are two natural generators of $G$. Therefore $c_{1}$, in general, describes the signature of a fiber bundle with 2-dimensional base space. Actually, it can be interpreted as a first Chern class [7].

Problem 8. Find the higher dimensional analogues of $c_{1}$ in $H^{2 q}(G, \mathbf{Z})$.

\section{The eta-invariant of the variation structures $([14,13])$}

Definition. Let $\mathcal{V}$ be an $\epsilon$-hermitian variation structure. Then for any $g \in G, \rho(g)$ determines a spectral decomposition

$$
(U ; b, \rho(g), V(g))=\bigoplus_{\chi}\left(U_{\chi} ; b_{\chi}, \rho(g)_{\chi}, V(g)_{\chi}\right),
$$

where $U_{\chi}$ is the generalized $\chi$-eigenspace of $\rho(g)$. The eta-invariant $\eta_{\mathcal{V}}(g)$ is defined by the $\operatorname{sum} \sum_{\chi} \eta_{\mathcal{V}}(g)_{\chi}$, where

$$
\eta_{\mathcal{V}}(g)_{\chi}= \begin{cases}(1-2 c) \operatorname{sign} b_{\chi} & \text { if } \chi=e^{2 \pi i c}, 0<c<1 \\ -\operatorname{sign}\left[\left(1+\rho(g)_{\chi}^{-1}\right) V(g)_{\chi}\right] & \text { if } \chi=1\end{cases}
$$

Our $\eta$-invariant can be interpreted as the $\eta$-invariant of the signature operator of the circle twisted with the signature bundle of a hermitian flat bundle, in the sense of [3].

a) Wall's cocycle via eta invariant

THEOREM. If $G$ is a (finitely generated) abelian group then

$$
\sigma\left(\mathcal{V} ; K_{e}, K_{g}, K_{h}\right)=-\eta(g)-\eta\left(g^{-1} h\right)+\eta(h) .
$$

In particular $\delta(\eta)=-\sigma$. 
Problem 9. Characterize variation structures $\mathcal{V}$ (or groups $G$ ) with the property $\delta(\eta)=-\sigma$.

b) The signature via Wall's cocycle and eta-invariant. Let $f=p \circ \phi$ as in the introduction. Let $\mathcal{V}(\phi)$ be the variation structure associated with $\phi$ as in Example 5.

Our goal is to compute $\sigma(f)$. For simplicity, assume that $p$ is reduced and irreducible.

Consider the splice diagram $\Gamma(p, \Delta)$ of the multilink determined by $p^{-1}(0) \cup \Delta \subset$ $\left(\mathbf{C}^{2}, 0\right)$, where the multiplicity of $p^{-1}(0)$ is 1 , and the other multiplicities are zero. Each node of the diagram represents a Seifert component $\Sigma\left(a_{1}, \ldots, a_{r} ; S_{1}, \ldots, S_{r}\right)$ with multilink $\left(\Sigma ; S_{1}, \ldots, S_{r} ; m_{1}, \ldots, m_{r}\right)$. Notice that the multiplicities $m_{i}$ can be determined from $\Gamma(p, \Delta)$ by the corresponding splicing conditions. Let $M_{i}$ resp. $L_{i}$ be the topological standard meridian resp. longitude of the link component $S_{i}$. Consider the following numbers: $q_{i}=a_{1} a_{2} \cdots a_{r} / a_{i}, m_{i}^{\prime}=-\sum_{j \neq i} m_{j} q_{j} / a_{i}$, and $n_{i}=\operatorname{gcd}\left(m_{i}, m_{i}^{\prime}\right)>0, i=1, \ldots, r$.

Recall that the quotient $\Sigma / S^{1}=B$ of $\Sigma$ by its free $S^{1}$-action is an $r$-punctured 2-sphere.

THEOREM ([13]). The signature $\sigma(f)$ is a sum $\sigma(f)=\sum_{\text {nodes }} \sigma(\Sigma, \rho)$ over the nodes of $\Gamma(p, \Delta)$, where:

(a) For a Seifert component $\Sigma$, the term $\sigma(\Sigma, \rho)$ is the signature of a hermitian flat bundle over the Milnor fiber of $\Sigma$ (or alternatively, over the r-punctured 2-sphere). Actually, it can be computed as a sum of Wall's cocycles associated with $\mathcal{V}(\phi)$.

(b) If the variation structure above $\Sigma$ is abelian, then

$$
\sigma(\Sigma, \rho)=\sum_{i=1}^{r} n_{i} \cdot \eta\left(L_{i}^{m_{i} / n_{i}} M_{i}^{m_{i}^{\prime} / n_{i}}\right) .
$$

c) An application: Yomdin's series

TheOREM. Let $f_{1}:\left(\mathbf{C}^{n+1}, 0\right) \rightarrow(\mathbf{C}, 0)$ be a germ with one-dimensional critical locus. Choose a germ $f_{2}$ in such a way that the pair $\phi=\left(f_{1}, f_{2}\right)$ is an ICIS. Then

$$
\sigma\left(f_{1}+f_{2}^{q}\right)-\sigma\left(f_{1}\right)=-q \eta(M)-\eta(L)+\eta(L+q M),
$$

where $q \gg 0$ and $L$ resp. $M$ are the standard topological longitude and meridian associated with the series $\left\{f_{1}+f_{2}^{q}\right\}_{q \gg 0}$.

(The definition of $L$ and $M$ is the following: let $\Delta_{0}$ be the image $\phi\left(\operatorname{Sing} f^{-1}(0)\right)$ of the singular locus of $f^{-1}(0)$. Consider the set $U_{P} \backslash E$ as in Problem 4. Its fundamental group is generated by a small circle (in $U_{P} \backslash E$ ) around $E$, called $L$, and a small circle around $\tilde{\Delta_{0}}$, called $M$.)

An interesting consequence is the quasi-periodicity property (which generalizes the corresponding result for the suspension case, conjectured by Brieskorn, Durfee and Zagier and proved by Neumann), namely, the function $q \rightarrow \sigma\left(f_{1}+f_{2}^{q}\right)$ is a sum of a linear function and some periodic functions.

We can consider here an even more particular case: the suspension case.

Let $f:\left(\mathbf{C}^{n}, 0\right) \rightarrow(\mathbf{C}, 0)$ be an isolated singularity. Consider $f_{1}:\left(\mathbf{C}^{n+1}, 0\right) \rightarrow(\mathbf{C}, 0)$ defined by $f_{1}\left(z, z_{n+1}\right)=f(z)$. Set $f_{2}=z_{n+1}$ and $\phi=\left(f_{1}, f_{2}\right)$ as above. The singular 
locus of the ICIS $\phi$ is $\Sigma=\{z=0\}$, and the discriminant locus $\Delta$ contains only one irreducible component $\Delta=\Delta_{0}$, which is smooth. In particular, $G=\mathbf{Z}$ and $L=0$. By the above theorem,

$$
\sigma\left(f+z_{n+1}^{q}\right)=\eta_{\mathcal{V}(f)}(q)-q \cdot \eta_{\mathcal{V}(f)}(1),
$$

where $\eta_{\mathcal{V}(f)}(q)$ is the eta-invariant of the variation structure $\mathcal{V}(f)$ (cf. Example 4) and $q \in \mathbf{Z}=G$.

Problem 10. Characterize composed germs $f=p \circ \phi$ such that $\sigma(f)$ can be computed only in terms of $\eta_{\mathcal{V}(\phi)}$ and $p$.

d) The eta-invariant and Hodge invariants. Let $s(\lambda)=0$ if $\lambda \neq 1$ and $s(\lambda)=1$ if $\lambda=1$. Denote the dimensions of the primitive spaces of the mixed Hodge structure of the germ $f$ by $p_{\lambda}^{p, q}(f)$ (where $r=p+q-n-s(\lambda) \geq 0$ ) (cf. $\S 4$ ). Consider the invariant $\Sigma p p_{\lambda, \pm}(f)=\sum(-1)^{q} p_{\lambda}^{p, q}$, where the sum is over the pairs $(p, q)$ so that $r=p+q-n-s(\lambda)$ satisfies $(-1)^{r}= \pm 1$. (Recall that $(r+1)$ measures the sizes of the Jordan blocks of the monodromy operator.)

THEOREM.

$$
\eta(\mathcal{V}(f) ; a)=-\sum_{\substack{\lambda^{a}=1 \\
\lambda \neq 1}} \Sigma p p_{\lambda,-}(f)-\sum_{\begin{array}{c}
\lambda^{a} \neq 1 \\
\text { or } \lambda=1
\end{array}}(1-2\{c a\}) \Sigma p p_{\lambda,+}(f) .
$$

Problem 11. Compute the eta-invariant of the variation structure described in problem $4 \mathrm{~b}$ in terms of the natural limit mixed Hodge structure (cf. problem 5).

\section{References}

[1] V. I. Arnold, S. M. Gusein-Zade and A. N. Varchenko, Singularities of Differentiable Mappings, Vol. 2, Birkhäuser, Boston, 1988.

[2] M. Demazure, Classification des germes à point critique isolé et à nombre de modules 0 ou 1 (d'après Arnold), Sém. Bourbaki $26^{\mathrm{e}}$ année, 1973/74, 443, (1974).

[3] M. F. Atiyah, V. K. Patodi and I. M. Singer, Spectral asymmetry and Riemannian geometry. I-III, Math. Proc. Cambridge Philos. Soc. 77 (1975), 53-69; 78 (1975), 405-432; 79 (1976), 71-99.

[4] A. Durfee and L. Kaufman, Periodicity of branched cyclic covers, Math. Ann. 218 (1975), 157-174.

[5] M. G. M. van Doorn and J. H. M. Steenbrink, A supplement of the monodromy theorem, Abh. Math. Sem. Univ. Hamburg 59 (1989), 225-233.

[6] J. Harer, The second cohomology group of the mapping class group of an orientable surface, Invent. Math. 72 (1983), 221-239.

[7] W. Meyer, Die Signatur von lokalen Koeffizientensystemen und Faserbundeln, Bonner Math. Schriften 53 (1972).

[8] J. Milnor, On isometries of inner product spaces, Invent. Math. 8 (1969), 83-97.

[9] A. Némethi, Generalized local and global Sebastiani-Thom type theorems, Compositio Math. 80 (1991), 1-14.

[10] —, The zeta function of singularities, J. Algebraic Geom. 2 (1993), 1-23. 
[11] A. Némethi, The real Seifert form and the spectral pairs of isolated hypersurface singularities, Compositio Math. 98 (1995), 23-41.

[12] - The semi-ring structure and the spectral pairs of sesqui-linear forms, Algebra Colloq. 1 (1994), 85-95.

[13] - The equivariant signature of hypersurface singularities and eta-invariant, Topology, 34 (2) (1995), 243-259.

[14] —, The eta-invariant of variation structures, Topology and its Applications, to appear.

[15] A. Némethi and J. H. M. Steenbrink, Spectral pairs, mixed Hodge modules and series of plane curve singularities, Dept. Math., Univ. of Nijmegen, Report, 1994; New York J. Math., August 16, 1995 (http://nyjm.albany.edu:8000/j/v1/Nemethi-Steenbrink.html).

[16] W. D. Neumann, Invariants of plane curve singularities, Monographie $N^{\circ} 31$ de L'Enseignement Math., 1983.

[17] R. Schrauwen, Topological series of isolated plane curve singularities, Eiseign. Math. 36 (1990), 115-141.

[18] J. H. M. Steenbrink, Mixed Hodge Structures on the Vanishing Cohomology, Nordic Summer School/NAVF, Symposium in Math., Oslo, 1976,

[19] C. T. C. Wall, Non-additivity of the signature, Invent. Math. 7 (1969), 269-274. 